\title{
MEDICIÓN DE LOS RESULTADOS ESTÉTICOS Y FUNCIONALES DESDE LA PERSPECTIVA DEL PACIENTE EN RINOPLASTÍA: CREACIÓN DEL INSTRUMENTO NOSE-QoL ${ }^{\circledR *}$
}

\author{
Drs. Stefan Danilla E. ${ }^{1}$, Marco Antonio Ríos V. ${ }^{1}$, Pedro Cuevas T. ${ }^{1}$, \\ María Elsa Calderón G. ${ }^{1}$, Carlos Domínguez C. ${ }^{1}$, Soc. Cristina Di Silvestre P. ${ }^{2}$, \\ Al. Paula Silva R. ${ }^{3}$, Al. Sofía Serra D. ${ }^{3}$, Drs. Patricio Andrades C. ${ }^{1}$, Cristian Erazo C. ${ }^{1}$, \\ Susana Benítez S. ${ }^{1}$, Rolando Shulz R. ${ }^{1}$, Jason Wasiak ${ }^{4}$, Sergio Sepúlveda P. ${ }^{1}$
}

1 Unidad de Cirugía Plástica, Departamento de Cirugía, Hospital Clínico de la Universidad de Chile, Santiago, Chile. 2 Socióloga Facultad de Medicina, Dirección de Postgrado, Universidad del Desarrollo, Clínica Alemana.

3 Alumnos de Medicina Universidad de Chile, Santiago, Chile.

4 Victorian Adult Burns Service and School of Public Health and Preventative Medicine, Monash University, The Alfred Hospital, Melbourne, Australia.

\begin{abstract}
Development of a new patient-reported outcome measure for rhinoplasty suergery: the Nose-QoL

Background: Instruments that assess the results of plastic surgery from patient's perspective allow a multidimensional evaluation of surgical results. Aim: To develop a new instrument to assess the functional and esthetic results of rhinoplasty from the point of view of the patient. Material and Methods: A conceptual framework about important results for nasal image was developed using information from in depth interviews to patients, focal groups with experts and interviews to esthetic surgeons and patients subjected to rhinoplasty. Results: Four patients and four plastic surgeons were interviewed. The three sub scales identified for the conceptual framework were quality of life, postoperative disability and pain and surgeon's objective evaluation scale. Five domains for quality of life were identified including nose size and form, self-image and esteem, third party perceptions and social relationships, nasal function and segmental nasal analysis. Conclusions: After completion of the psychometric assessment, the Nose-QoL instrument will become a valuable instrument to measure the impact and effectiveness of rhinoplasty from the patient's perspective.
\end{abstract}

Key words: Rhinoplasty, patient satisfaction, quality of life.

\section{Resumen}

Objetivo: Desarrollar un nuevo instrumento de resultados estéticos y funcionales desde la perspectiva del paciente, diseñado para medir la satisfacción de éstos en relación a rinoplastía. Métodos: Revisión de

*Recibido el 21 de junio de 2013 y aceptado para publicación el 5 de julio de 2013.

Declaración: Ninguno de los autores tiene conflicto de intereses potencial de revelar.

Correspondencia: Dr. Stefan Danilla E.

Santos Dumont 999, Santiago, Chile. CP 8380456

drstefandanilla@gmail.com 
la literatura, entrevistas en profundidad a pacientes, grupos focales de discusión de expertos, a cirujanos plásticos y pacientes sometidos a rinoplastías, con lo que se desarrolló un marco conceptual en relación a los resultados considerados importantes para la imagen nasal. Resultados: Se entrevistó a cuatro pacientes y cuatro cirujanos plásticos. Las tres Subescalas identificadas para el marco conceptual incluyen: la Calidad de Vida (CdV), la Escala de Discapacidad y Dolor Post-operatorio (EDDP) y Escala de Evaluación Objetiva del Cirujano (EEOC). Se identificaron 5 dominios para la subescala CdV, que incluyen: tamaño y forma de la nariz, autoimagen y autoestima, percepción de terceros y relaciones sociales, función nasal y análisis nasal segmentario. Conclusiones: Una vez completada la evaluación psicométrica, el instrumento Nose-QoL y subescalas, proporcionará una herramienta confiable para cirujanos plásticos, investigadores y pacientes para medir el impacto y la eficacia de las rinoplastías, desde la perspectiva del paciente. Nivel de Evidencia: $1-$ Estudio cualitativo, generación de instrumentos de medición de resultado desde la perspectiva del paciente.

Palabras clave: Cirugía nasal estética, medicina basada en la evidencia, cuestionario psicométrico, encuesta, satisfacción del paciente.

\section{Introducción}

Desde el inicio del desarrollo de la medicina basada en la evidencia (MBE), a mediados de la década de los ' $70 \mathrm{~s}^{1,2}$, la medicina clínica ha experimentado una explosión en la generación de conocimientos, mejorando así la calidad en distintas áreas de atención al paciente ${ }^{3}$. Actualmente, los estudios de cohorte adecuadamente diseñados, así como los ensayos aleatorizados controlados constituyen el estándar para la adquisición del conocimiento de la historia natural de las enfermedades o la determinación de la eficacia y efectividad de nuevos tratamientos, como también de los ya existentes ${ }^{4}$.

En la cirugía estética y funcional de la nariz, las preocupaciones del cirujano son principalmente una forma adecuada del dorso y de la punta nasal, líneas adecuadas de osteotomía, con o sin open roof, presencia y ausencia de injertos de cartílago visibles, la septoplastía como procedimiento asociado en el caso de síntomas funcionales o desviaciones antiestéticas; todo con el objetivo de obtener un mejor resultado estético y funcional para el paciente. Con el tiempo, la aparición de nuevas técnicas y la sistematización de las existentes, ha llevado a la popularización de ellas y a la aparición de un sinúmero de variantes que presumen mejorar el resultado estético.

Generalmente, los resultados de cirugías cosméticas son presentados en publicaciones científicas como fotografías de antes y después, y hasta hace muy poco, no existían instrumentos desarrollados para medir los resultados desde la perspectiva del paciente.

Los instrumentos de evaluación de resultado desde la perspectiva del paciente, evalúan los resultados de la cirugía en una escala multidimensional, no sólo evaluando el parámetro estético, sino también la mejora en el bienestar físico, psicológico y social después de la cirugía. La medición de resultados desde la perspectiva del paciente son cuestionarios altamente sofisticados que cuantifican la calidad de vida relacionada a la salud y otras variables importantes desde esta perspectiva.

Nuestro grupo tradujo al español y validó el instrumento BREAST- $Q$ (Pusic y cols) ${ }^{5}$, diseñado para medir la calidad de vida relacionada a cirugía de $\mathrm{mama}^{6}$, encontrando resultados altamente confiables en nuestra población. También hemos desarrollado la escala preliminar del instrumento BODY- $Q^{7}$ diseñado para medir la satisfacción de los pacientes en relación a los procedimientos de contorno corporal como la liposucción, abdominoplastía, lifting corporal, levantamiento de muslos y pexia de brazos.

El objetivo de nuestro trabajo fue el desarrollo de un nuevo instrumento de resultados desde la perspectiva del paciente llamado Nose-QoL, separado en un conjunto de subescalas como: imagen nasal, armonía facial, tamaño y forma de la nariz, auto-imagen y auto-autoestima, relaciones sociales, síntomas físicos, etc. (Figura 1).

Estas subescalas están diseñadas para medir una serie de resultados que hemos identificado como importantes para los pacientes sometidos a rinoplastía.

\section{Métodos}

Se siguieron guías internacionales de confección de instrumentos de evaluación de resultados desde la perspectiva del paciente para desarrollar el contenido de las escalas de cirugía de contorno corporal $^{8,9}$. En términos generales, un instrumento de evaluación de resultados desde la perspectiva del paciente, válido y confiable estadísticamente, debe ser desarrollado en tres fases: 1) marco conceptual y desarrollo preliminar de las escalas; 2) reducción de ítems y evaluación psicométrica y 3 ) prueba final del instrumento (Tabla 1).

El presente reporte corresponde a la primera fase 


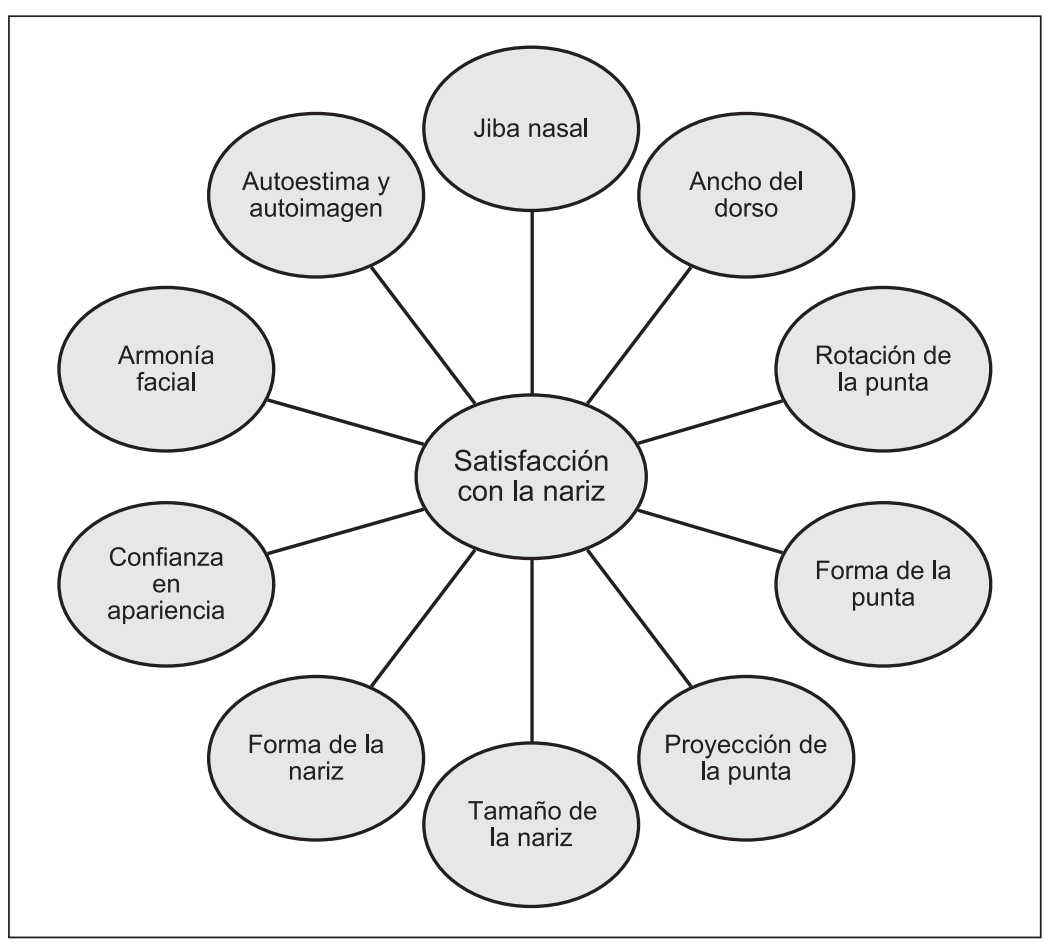

Figura 1. Marco conceptual sobre la satisfacción con la apariencia y función nasal.

Tabla 1. Fases de desarrollo de la medición de los resultados desde la perspectiva del paciente

\begin{tabular}{|ll|}
\hline Fase I & $\begin{array}{l}\text { Construcción de un marco conceptual, donde se incluyan todas las dimensiones del fenómeno a medir. Se de- } \\
\text { sarrolla mediante una entrevista semi-estructurada realizada por un sociólogo entrenado, junto con la opinión } \\
\text { de expertos y la revisión de la literatura. El instrumento preliminar se prueba en una muestra de pacientes para } \\
\text { aclarar ambigüedades en la redacción de artículos, confirmar la pertinencia y determinar la aceptabilidad y el } \\
\text { tiempo total para completar el cuestionario }\end{array}$ \\
Fase II & $\begin{array}{l}\text { El cuestionario se aplica a una muestra amplia de pacientes para elegir los mejores ítems para su inclusión en } \\
\text { el instrumento final. En esta fase, la evaluación psicométrica se lleva a cabo y la escala se reduce filtrando el } \\
\text { cuestionario, para desarrollar un instrumento corto con los mejores ítems }\end{array}$ \\
Fase III $\quad \begin{array}{l}\text { Se lleva a cabo la evaluación psicométrica del instrumento final, incluyendo el coeficiente de confiabilidad, } \\
\text { selección, validez, sensibilidad, especificidad, la correlación total ítems, etc. El objetivo es determinar las for- } \\
\text { talezas y limitaciones del instrumento desarrollado }\end{array}$
\end{tabular}

de nuestro estudio. Nuestra meta fue generar un marco conceptual para la generación de dominios e ítems, realizando una revisión exhaustiva de la literatura, discusión de grupos de expertos y entrevistas en profundidad a pacientes.

Utilizando un diseño cualitativo, realizamos entrevistas en profundidad con informantes clave. Los informantes clave fueron pacientes programados para rinoplastía, incluyendo rinoplastías con o sin septoplastía, con fines estéticos, funcionales o ambos.

Se realizaron entrevistas semi estructuradas por una socióloga entrenada (Master of Science en Epidemiología Clínica con vasta experiencia en investigación cualitativa); durante la entrevista, se permitió a los pacientes hablar libremente sobre sus motivaciones para realizar la cirugía, además fueron interrogados específicamente sobre conceptos de belleza en relación a la imagen nasal y armonía facial, tamaño y forma de la nariz, autoimagen y autoestima, relaciones sociales y síntomas funcionales (Tabla 2). Cada entrevista duró aproximadamente una hora; posteriormente, los pacientes fueron entrevistados tres meses luego de la cirugía, confrontando 
Tabla 2. Guía para la entrevista en profundidad semi-estructurada

Razones para la cirugía: Influencia/opinión/percepción de la pareja, los amigos, familia y/o de la sociedad, la motivación, tipo de procedimiento elegido

Imagen nasal y armonía facial: Apariencia facial en general, la armonía de la cara

Tamaño y forma nasal: Tamaño de la giba nasal, proyección y alineamiento de la punta nasal, desviaciones nasales

Auto imagen corporal: Conexión con otras personas, confianza en sí mismo, autoestima

Relaciones sociales: Relaciones interpersonales con colegas del trabajo y vida cotidiana, impacto en los amigos

Síntomas funcionales: Presencia de dolor nasal, dificultad para respirar u obstrucción nasal por desviación del tabique, sensación de mal olor constante, secreción nasal

Tabla 3. Características básicas de los pacientes y cirujanos entrevistados

\begin{tabular}{|lc|}
\hline Características & $\begin{array}{c}\text { Pacientes entrevistados } \\
(\mathbf{n}=\mathbf{4})\end{array}$ \\
$\begin{array}{l}\text { Edad, años } \\
\text { Promedio } \pm \text { desviación estándar }\end{array}$ & 35 años $\pm 4,2$ \\
Rango & 30 a 40 años \\
Género & \\
$\quad$ Femenino & 3 \\
$\quad$ Masculino & 1 \\
Tipo de cirugía & \\
$\quad$ Rinoplastía sola & 3 \\
$\quad$ Rinoplastía + Septoplastía & 1 \\
Características & Cirujanos entrevistados \\
Promedio años de práctica & (n= 4) \\
profesional & 9,2 años $\pm 5,7$ \\
Rango & 3 a 18 años \\
\hline
\end{tabular}

las creencias previas con la situación actual tras la cirugía.

Además, se realizaron entrevistas en profundidad a cirujanos plásticos con experiencia en al menos 20 rinoplastías y representativos de prácica junior, intermedia y senior.

El muestreo se llevó a cabo hasta la saturación de la información, de acuerdo a los estándares cualitativos de investigación: cuando los nuevos pacientes entrevistados no aportaron mayor información de contenido que la ya recolectada, finalizamos el muestreo.

Las entrevistas fueron transcritas; luego, las afirmaciones significantes obtenidas de las transcripciones se agruparon en un agregado de temas comunes. Se desarrollaron ítems a partir de las afirmaciones significantes. Los ítems generados fueron agrupados en dominios surgidos de los agregados de temas comunes, construyendo la escala preliminar.

\section{Resultados}

Un total de 4 pacientes ( 3 mujeres y 1 hombre) y 4 cirujanos plásticos fueron entrevistados desde el 10 de julio de 2012 hasta el 30 de abril de 2013. Las características de los pacientes se muestran en la Tabla 3.

De las entrevistas a pacientes, se identificaron 116 afirmaciones significantes que se subdividieron en 2 subescalas: Calidad de Vida (CdV) y la Escala de Discapacidad y Dolor Post-operatorio (EDDP). Se identificaron 5 dominios y 78 ítems para la subescala $\mathrm{CdV}$. Un ejemplo del proceso de generación de un ítem a partir del proceso de entrevista se grafica en la Figura 2. Un esquema de la subescala $\mathrm{CdV}$, sus respectivos dominios e ítems para el desarrollo del instrumento NoseQoL, se representa en la Figura 3.

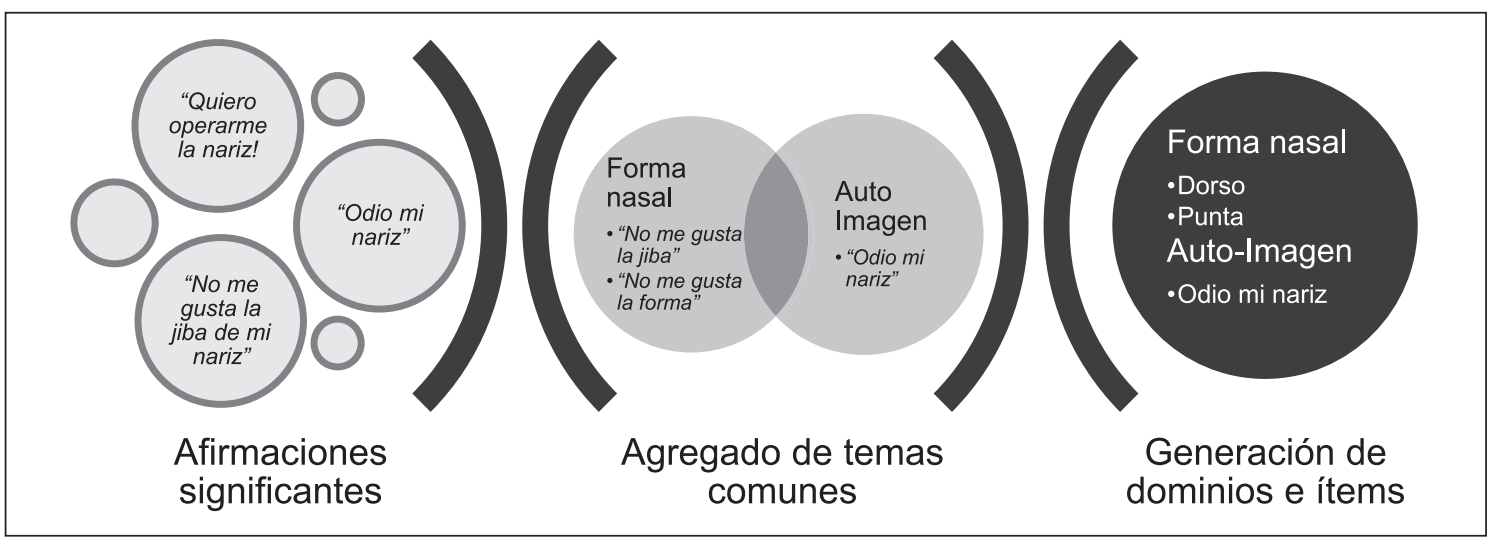

Figura 2. Representación esquemática de la generación de ítems y dominios. 


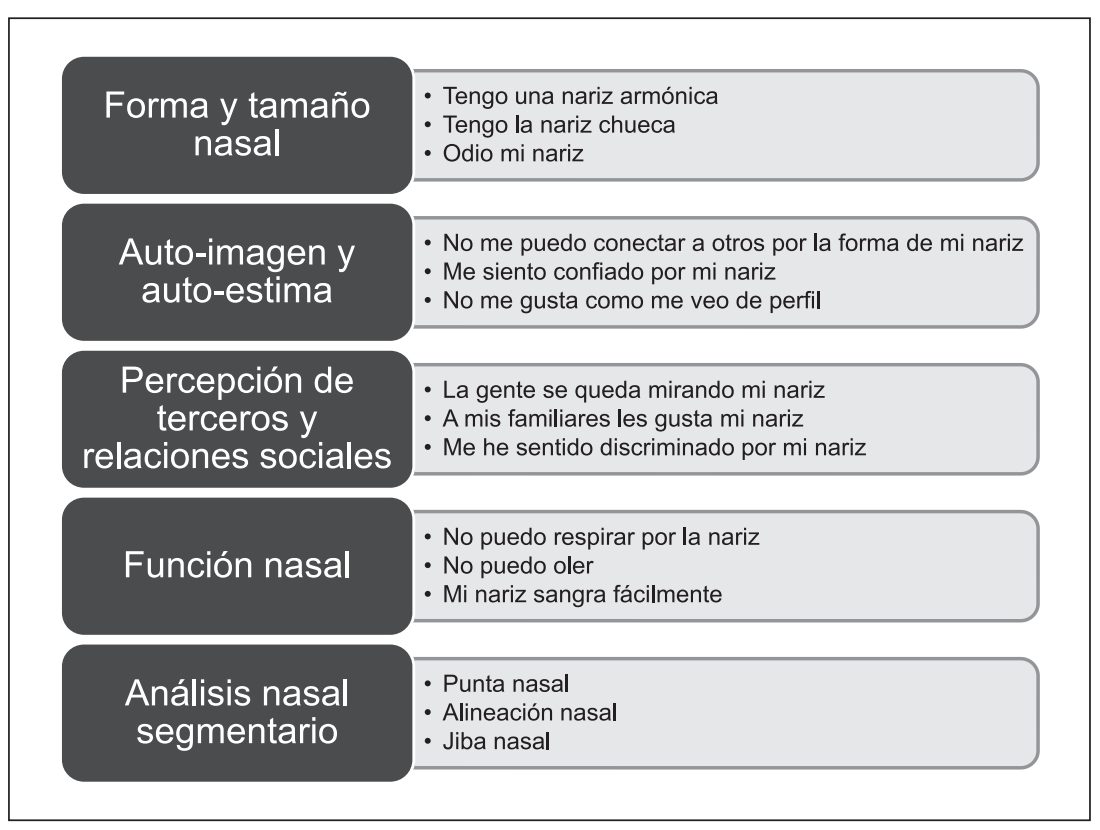

Figura 3. Agregado de ítems y dominios.

\section{Calidad de vida}

- Forma general de la nariz - Auto imagen y autoestima

- Percepción de terceros

- Función nasal

- Análisis nasal segmentario

\section{Escala de dolor y} discapacidad postoperatoria

- Dolor

- Inflamación

- Dificultad para respirar

- Dificultad para oler

- Dificultad para comer

\section{Evaluación técnica del} cirujano

- Definición de la punta

- Deformidad supratip

- Dorso

- Deformidad en V invertida

- Visibilidad de injertos

Figura 4. Composición del instrumento Nose-QoL.

Además 22 ítems fueron identificados para la subescala EDDP, conformando el instrumento preliminar. En el mismo período, se entrevistó a cuatro cirujanos plásticos. Las características de los cirujanos se muestran en la Tabla 3. De estas entrevistas, se identificaron 37 afirmaciones significantes que se agruparon en la Escala de Evaluación Objetiva del Cirujano (EEOC) arrojando 22 ítems.

Los ítems fueron organizados como afirmaciones en escala de Likert de cinco puntos, en donde el paciente expresa su acuerdo con la afirmación desde "completamente de acuerdo" hasta "completamente en desacuerdo". En la Tabla 4 se muestra un ejemplo de las 3 subescalas, los dominios del $\mathrm{CdV}$ e ítems.

Por lo tanto, tres módulos representados esquemáticamente en la Figura 4 componen el instrumento preliminar Nose-QoL.

\section{Conclusiones}

La capacidad de medir la satisfacción del paciente con procedimientos quirúrgicos ha aumentado en importancia ${ }^{8,9}$. Desde un punto de vista académico, nos permite cuantificar objetivamente los resultados de una determinada técnica, así como 
Tabla 4. Ejemplo de las tres subescalas mayores; los cinco dominios de la subescala "Calidad de Vida" y sus respectivos ítems; correspondientes al instrumento preliminar Nose-QoL

\begin{tabular}{|l}
\hline Subescalas, Dominios e ítems \\
Calidad de vida \\
Tamaño y forma de la nariz (13 items) \\
Tengo una cara armónica \\
Tengo la nariz chueca \\
Odio mi nariz \\
Autoimagen y autoestima (30 items) \\
No me puedo conectar con otras personas por mi nariz \\
Mi nariz me da confianza \\
No me gusta mi perfil \\
Percepción de terceros y relaciones sociales (10 items) \\
Siento que la gente mira mi nariz \\
A mis familiares les gusta mi nariz \\
Me he sentido discriminado por mi nariz \\
Función Nasal (18 items) \\
No puedo respirar por la nariz \\
No siento olores \\
Sangro fácilmente por la nariz \\
Análisis nasal segmentario (7 items) \\
Punta nasal \\
Alineamiento nasal \\
Jiba \\
Escala de discapacidad y dolor post-operatorio EDDP (22 ítems) \\
Siempre siento un mal olor en mi nariz \\
Tengo la nariz hinchada \\
No puedo comer porque me siento atorado \\
Escala de evaluación objetiva del cirujano EEOC (22 ítems) \\
Injertos visibles \\
Apariencia natural \\
Presencia de supratip
\end{tabular}

también comparar entre técnicas ${ }^{10-12}$. Además, desde una perspectiva clínica, nos permite mejorar la relación cirujano-paciente, mostrándole al paciente su mejora, haciéndolos partícipes de su proceso de recuperación ${ }^{11,12}$.

En la primera fase de nuestro estudio, las entrevistas realizadas nos permitieron recolectar información valiosa, con lo que se pudo desarrollar la escala preliminar.

Los dominios desarrollados fueron consistentes y coherentes con el conocimiento previo y con publicaciones anteriores ${ }^{13,14}$.

El Nose-QoL constituirá un instrumento robusto y completo, con 3 subescalas para los procedimientos asociados a rinoplastías: CdV, EDDP y EEOC.

Una vez que las tres fases estén completas, la versión final del instrumento Nose-QoL nos permitirá identificar "valores normales" para la población, medidas de la satisfacción del paciente con los procedimientos asociados a rinoplastías, el impacto de estos procedimientos en la calidad de vida del paciente, y comparar entre distintas técnicas quirúrgicas o cirujanos.

Nuestro próximo reporte será luego de concluida las fases 2 y 3 de nuestro estudio, las que se encuentran en ejecución actualmente. 


\section{Referencias}

1. Zimerman Al. Evidence-based medicine: a short history of a modern medical movement. Virtual Mentor 2013; 15:71-6.

2. Jeffrey A, Timothy C. History and Development of Evidence-based Medicine. World Journal of Surgery 2005;29:547-53.

3. Manary MP, Boulding W, Staelin R, Glickman SWN. The Patient Experience and Health Outcomes. Engl J Med. 2013;368:201-3.

4. Sackett DL, Straus S, Richardson WS, Rosenberg W, Haynes B. Evidence-Based Medicine: How to Practice and Teach EBM. 2000. 2nd ed. Edinburgh \& New York: Churchill Livingstone.

5. Pusic AL, Klassen AF, Scott AM, Klok JA, Cordeiro PG, Cano SJ. Development of a new patient-reported outcome measure for breast surgery: the BREAST-Q. Plast Reconstr Surg. 2009;124:345-53.

6. Cuevas P, Calderón M, Erazo C, Benítez S, Andrades P, Sepúlveda S y col. Mamoplastía de reducción: resultados desde la perspectiva del paciente. Validación lingüística y psicométrica del Breast Q Reduction and Mastopexy Module Instrument. Rev Chil Cir. 2013;65:146-9.

7. Measuring Patient-Reported Outcomes in Body Contouring Patients: Development of the Body-Q. (In Press).

8. Kazis L, Anderson J, Meenan R. Effect sizes for interpreting changes in health status. Med Care 1989;27:178-
89.

9. Ramsey A, Larrabee W, Anderson S, Craig M, Calvin M. Measuring Cosmetic Facial Plastic Surgery Outcomes. Arch Facial Plast Surg. 2001;3:198-201.

10. Cano S, Klassen A, Pusic A. The science behind qualityof- life measurement: A primer for plastic surgeons. Plastic and Reconstructive Surg. 2009;123:98-106.

11. Thoma A, Veltri K, Khuthaila D, Rockwell G, Duku E. Comparison of the deep inferior epigastric perforator flap and free transverse rectus abdominis myocutaneous flap in postmastectomy reconstruction: A costeffectiveness analysis. Plastic and Reconstructive Surg. 2004;113:1650-61.

12. Efficace F, Bottomley A, Osoba D, Gotay C, Flechtner $\mathrm{H}$, D'haese $\mathrm{S}$, et al. Beyond the development of healthrelated quality-of-life (HRQOL) measures: A checklist for evaluating HRQOL outcomes in cancer clinical trials. Does HRQOL evaluation in prostate cancer research inform clinical decision making? J Clin Oncol. 2003;21:3502-11.

13. Klassen AF, Cano SJ, Scott A, Snell L, Pusic AL. Measuring patient-reported outcomes in facial aesthetic patients: development of the FACE-Q. cial Plast Surg. 2010;26303-9. doi: 10.1055/s-0030-1262313. Epub 2010 Jul 27.

14. Pusic AL, Chen CM, Cano S, Klassen A, McCarthy C, Collins ED, et al. Measuring quality of life in cosmetic and reconstructive breast surgery: a systematic review of patient-reported outcomes instruments. Plast Reconstr Surg. 2007;120:823-37; discussion 838-9. 